\title{
Evaluation of Genetic Renal Diseases in Potential Living Kidney Donors
}

\author{
S. Kuppachi • R. J. H Smith • C. P. Thomas
}

Published online: 15 January 2015

(C) Springer International Publishing AG (outside the USA) 2015

\begin{abstract}
Better outcomes for living donor transplantation, together with the shortened waiting time and the increasing gap between supply and demand for kidneys for transplantation, has led to greater pressure on transplant candidates and transplant centers alike to encourage and advocate for living kidney donation as the more timely form of renal replacement therapy. Recent studies report that living kidney donors biologically related to their transplant candidate may be at increased risk of end-stage renal disease (ESRD) and this may reflect the shared inheritance of a disease or risk allele. The possibility of carrying a genetic predisposition or the renal disease itself should be entertained in every kidney donor with
\end{abstract}

This article is part of the Topical Collection on Live Kidney Donation

S. Kuppachi • R. J. H. Smith • C. P. Thomas

Department of Internal Medicine, University of Iowa College of

Medicine, Iowa City, IA, USA

\section{R. J. H. Smith • C. P. Thomas}

Department of Pediatrics, University of Iowa College of Medicine, Iowa City, IA, USA

\section{R. J. H. Smith}

Interdepartmental Program in Genetics, University of Iowa College of Medicine, Iowa City, IA, USA

\section{R. J. H. Smith • C. P. Thomas}

Interdepartmental Program in Molecular Cellular Biology, University of Iowa College of Medicine, Iowa City, IA, USA

R. J. H. Smith • C. P. Thomas

Rare Renal Disease Clinic, University of Iowa College of Medicine, Iowa City, IA, USA

\section{P. Thomas}

Veterans Affairs Medical Center, Iowa City, IA, USA

C. P. Thomas $(\bowtie)$

Organ Transplant Center, SE419 GH, University of Iowa Carver College of Medicine, 200 Hawkins Drive, Iowa City, IA 52242, USA

e-mail: christie-thomas@uiowa.edu a positive family history of kidney disease. Screening laboratory, imaging, and genetic tests have been established to assess early disease or future disease in individuals at risk for some genetic diseases. Ascertaining the cause of kidney disease in patients referred for a kidney transplant greatly facilitates appropriate screening for their genetically related potential living donor. However, there are a large number of monogenic renal diseases, from the common and easily diagnosed autosomal dominant polycystic kidney disease (ADPKD) to the rare and more difficult to recognize diseases that nevertheless pose a serious risk. In this review, we discuss approaches to screen for and exclude a few common renal genetic diseases including ADPKD, autosomal dominant interstitial kidney disease, some of the inherited podocytopathies, APOL1related renal disease, atypical hemolytic uremic syndrome, Alport syndrome, and Fabry disease.

Keywords Genetic testing - Genetic risk ·

Exome sequencing · Polycystic kidney disease .

Medullary cystic kidney disease $\cdot$ Interstitial disease .

Congenital nephrotic syndrome

Steroid-resistant nephrotic syndrome .

Focal and segmental glomerulosclerosis .

APOL1-related disease .

Atypical hemolytic uremic syndrome · Alport syndrome ·

Thin basement membrane nephropathy $\cdot$ Fabry disease

\section{Introduction}

The preferred therapy for end-stage renal disease (ESRD) is living donor kidney transplantation because it allows for timely transplantation, is associated with superior patient and graft 
outcomes in comparison with deceased donor transplantation and offers greatly improved survival compared with any form of chronic dialysis $[1,2,3 \cdot \bullet, 4 \bullet \bullet]$. The kidney transplant waiting list in the United States has grown from less than 10,000 in 1988 to over 100,000 in 2014, reflecting a growing unmet need for kidneys for transplantation.

Earlier studies of large single-center cohorts and US registry data reported that rates of ESRD in living kidney donors were lower than those of the general population among both White and African-American donors [1, 2, 3••]. However, more recent studies that better match living donors with population controls with similar risk factors report higher rates of ESRD in living donors than in these controls $[4 \bullet \bullet, 5 \bullet \cdot$. Biologically related donors form the largest group of living kidney donors, and it is thus important to assess and quantify, where possible, the genetic risk for kidney disease in these candidates as an important component of the living donor selection process. This paper discusses some of the more common known genetic risk factors for ESRD and the evaluation of genetic diseases in biologically related living donors.

\section{Importance of Establishing a Diagnosis in a Family Affected by Disease}

Determining the cause of ESRD in a transplant candidate is essential to establish the risk of recurrent disease after transplant in the kidney recipient and to determine the risk of serious renal disease for the biologically related donor.

According to the 2012 Scientific Registry of Transplant Recipients (SRTR) annual data report, the primary cause for ESRD in waitlisted patients was diabetes mellitus (DM) in $34.2 \%$, hypertension in $25.0 \%$, cystic kidney disease in $8.2 \%$, and other or unknown in $18.5 \%$ [6]. Diabetic nephropathy is often a clinical diagnosis made in diabetic ESRD patients without a kidney biopsy. In a retrospective analysis of renal biopsies performed in diabetic patients, $36 \%$ of patients had non-diabetic renal disease, and another $27 \%$ had diabetic renal disease in addition to non-diabetic renal disease [7]. In another study of diabetic patients with albuminuria and no retinopathy, non-diabetic glomerulopathies or a normal glomerular structure was seen in $31 \%$ [8]. Similarly, it is only seldom that a kidney biopsy is performed in those thought to have hypertension-causing ESRD. This may be partly because the initial presentation to a nephrologist is with advanced kidney disease, at which time a biopsy may be unable to establish the true etiology or is considered therapeutically futile. We now know that APOL1 gene variants are strongly associated with kidney disease that has historically been labeled 'hypertensive' or arteriolar nephrosclerosis in African-Americans [9॰]. We believe that a significant number of patients with ESRD and associated DM and/or hypertension do not have a clearly identified cause of ESRD. The absence of a diagnostic imaging study or diagnostic biopsy translates to an unclear risk of genetic kidney disease in biologically related kidney donors and underscores the importance of considering genetic kidney disease in every waitlisted transplant candidate who does not have a clearly established etiology of kidney disease.

\section{General Diagnostic Tools to Estimate Risk of Genetic Disease}

\section{Detailed Family History}

A detailed family history for kidney disease should be sought in every donor and in every transplant candidate who may have a biologically related donor. For autosomal-dominant diseases with a high degree of penetrance, such as autosomal dominant polycystic kidney disease (ADPKD) from mutations in $P K D 1$, having other affected family members is the norm, but $5-10 \%$ of the time the causal $P K D 1$ mutation arises de novo and hence a family history of ADPKD may be absent [10]. Other autosomal-dominant disorders, like renal cysts and diabetes (RCAD) syndrome, show remarkable variability in the phenotypic presentation or expressivity and limited penetrance. Consequently, renal manifestations may not be evident in some affected individuals and in others early-onset diabetes may present without renal disease [11].

With autosomal-recessive disorders such as the nephronophthisis or many childhood-onset steroid-resistant nephrotic syndromes, small nuclear families with no evidence of parental consanguinity may have just one affected individual, giving the appearance of a sporadic non-genetic disease. A pedigree of multigenerational disease with absent male-tomale transmission, where the more affected or only affected individuals are males with an apparent skipped generation is classic for X-linked disease. In some X-linked causes of ESRD, such as Fabry disease and Alport syndrome, while males are generally more severely affected, many obligate carrier females are not just asymptomatic 'carriers' but have or are at risk of developing advanced renal disease due to random X-chromosome inactivation.

\section{Linkage Testing}

The traditional method for establishing the genetic basis of disease involves linkage testing using a panel of highly informative microsatellite DNA markers flanking the putative affected genes, or in cases where there is locus heterogeneity or uncertainty about the nature of the genetic disease, using DNA markers that cover the whole genome. In large families with multiple affected individuals, it is possible to identify through statistical studies, DNA markers that are sufficiently close to the disease gene in question to 'co-segregate' together. The 
consequence of this close association is that every person with the disease phenotype under study (and hence the disease gene being sought) also has the closely linked genetic marker being followed. To successfully identify the location of the causal gene, linkage analysis requires large dominant families or consanguineous recessive families, a constraint that makes this strategy unsuitable for small pedigrees. There is now a wealth of knowledge detailing the genetic causes of many renal diseases, and newer testing methods make linkage testing unnecessary.

\section{Gene-Specific Mutation Detection}

In cases where a genetic disease is diagnosed clinically in a transplant candidate, and the list of causal genes is limited, direct sequencing of the affected gene is practical. For example, sequencing of the PKDl gene in a patient with ADPKD may identify the disease-causing genetic variant. Targeted mutation detection can then be undertaken in a biologically related donor candidate to determine whether the donor carries the ADPKD mutation. In practice, because $85 \%$ of known disease-causing mutations involve changes in the protein coding region, when a specific gene is selected for testing, the exons (those regions of the gene that encode the protein) of the candidate gene or genes is first amplified by polymerase chain reaction (PCR) and then sequenced to identify a possible causal mutation. While frameshift, nonsense, or splice site mutations are highly likely to be pathogenic, sometimes there is uncertainty in determining the significance of a sequence variation found in a gene of interest, such as a single amino acid substitution. In these situations, the sequence change is compared with the single nucleotide polymorphism database (dbSNP) and to disease-specific mutation databases (e.g. (http://pkdb.mayo.edu) to assess its prevalence in the general population and in patients affected with disease. The DNA sequence is also analyzed with various bioinformatic algorithms such as PolyPhen, MutationTaster, and the Grantham Matrix score to determine evolutionary conservation of the amino acid that is changed and to predict the pathogenic potential of the sequence change. Cases where no causal mutation is identified are particularly challenging and can reflect a non-genetic renal disease, a novel genetic cause of renal disease, or a missed genetic mutation in the sequenced gene because the mutation is outside the sequenced region or there is a large deletion or rearrangement of one allele that prevents amplification of the affected gene.

\section{Exome or Whole Genome Sequencing}

With the sequencing of the human genome and the development of high-throughput sequencing technologies it has become possible to sequence the entire human genome or the protein coding region of all genes (the exome) at low cost and in a very short period of time. The technology continues to evolve; however, in one method, the patient's DNA is broken into small fragments and hybridized to complementary sequences that cover the entire genome or the whole exome. The hybridized sequences are then captured ('hybrid capture'), amplified, and sequenced using massively parallel or Next Generation Sequencing (NGS) technology [12•, 13]. The power of the technology also allows an unbiased or semi-biased testing of a patient's DNA without any knowledge of where the genetic defect may lie. However, genetic variants are very common in the human genome or exome, and large amounts of sequence data are generated, requiring significant computational analysis and expertise to determine the significance of the variants identified. Nevertheless, this technology has led to the identification of a number of rare causes of genetic disease and has now expanded to the study of the epigenome and the study of somatic mutations in diseases like cancer.

A variation of whole exome sequencing is called targeted exome sequencing, where either a single gene or multiple genes of interest (e.g. the OtoSCOPE panel of genes that cause hereditary hearing loss, http://www.medicine.uiowa.edu/ morl/otoscope/) are captured from prepared DNA using complementary baits to the candidate genes and then sequenced by hybrid capture and NGS. This technique was used in the screening of PKD1 and PKD2 in a cohort of patients with ADPKD and in the screening of 40 candidate genes that cause early-onset inflammatory bowel disease (IBD) in a cohort of children with IBD [14, 15], reducing the cost and time for sequence analysis compared with the conventional techniques currently used. Several laboratories are developing targeted sequence capture platforms that will screen for a large number of renal diseases and will be useful for the relatively unbiased screening of living donors and their recipient candidates where the specific disease in the intended recipient has not been identified [16]. This testing technique will likely identify a number of variants that will need to be carefully considered to determine whether it might be causally related to the renal phenotype being investigated. Analysis of the genotyping data in the context of the patient's phenotypic data will be essential to make a final diagnosis.

\section{Specific Diseases}

\section{Autosomal-Dominant Polycystic Kidney Disease} (ADPKD)

ADPKD is the most commonly inherited renal disease. It is prevalent in 1 in 400-1,000 live births and accounts for more than $5 \%$ of ESRD in Europe and North America [17, 18]. ADPKD results from mutations in one of two genes, $P K D 1$ on chromosome 16p13 and $P K D 2$ on $4 \mathrm{q} 21$. In an assessment of a 
cohort of 202 probands, $P K D 1$ mutations accounted for $85 \%$ of cases and $P K D 2$ for $15 \%$ [19••], although other population-based studies have shown a higher frequency of $P K D 2$ mutations [20].

$P K D 1$ mutations are associated with a more aggressive clinical course, with ESRD developing at a median age of 54.3 years versus 74.0 years for PKD2 [21]. Diagnosis of ADPKD in people with a positive family history had been primarily based on the detection of renal cysts by ultrasound according to agebased criteria first published in 1994 and updated in 2009 to include populations at risk for ADPKD secondary to mutations in either PKD1 or PKD2 [22, 23]. According to these criteria, ADPKD can be excluded with high confidence in the absence of cysts for people between ages 30 and 39 (negative predictive value $[\mathrm{NPV}] 98.3 \%$ ), and in the presence of fewer than two cysts for patients $\geq 40$ years (NPV $100.0 \%$ ). Clearly, ultrasonography is limited in excluding a diagnosis in at risk individuals younger than 30 years, even in the absence of cysts (NPV $90.8 \%$ ), and a negative ultrasound does not exclude disease between the ages of 30 and 39 years in about $1.7 \%$ of those at risk. A more recent study compared non-contrast magnetic resonance imaging (MRI) and high-resolution ultrasound in a mixed cohort of 126 ADPKD patients (aged 16-40 years) and demonstrated an extremely high specificity and $100 \%$ sensitivity for MRI even in the group aged $<30$ years [24*0]. In this study, the presence of fewer than five cysts on MRI in both kidneys combined excluded the disease in individuals aged between 16 and 40 years with a family history of ADPKD. It is important to note that high-resolution ultrasound in this study had an improved sensitivity and NPV (98\%), even in the group aged 16-29 years compared with that previously published. But caution must be used in extrapolating this new ultrasound data as it is operator and equipment dependent, and it may have a lower sensitivity in detecting cysts in obese individuals.

\section{Testing of Living Donor Candidates}

Direct DNA sequencing is employed as the screening method for commercial genetic testing for ADPKD. The PKD1 gene consists of 46 exons adjacent to six homologous pseudogenes on chromosome 16p. The majority of the mutations are unique to single families. A large number of single amino acid substitutions have been identified that may not be disease related, and computational analysis is deployed to improve the prediction of the pathogenic effect of a single amino acid change. Furthermore, gene duplications, and gene rearrangements and large deletions can make amplification and sequencing difficult. Two large analyses suggest that definite or probable pathogenic mutations can be identified in about $89 \%$ of patients with a family history [19••, 25]. In those where no likely pathogenic mutation is identified, additional testing should be performed to look for large genetic rearrangements, and such testing is available in some commercial laboratories.
However, these events can be technically difficult to identify in the PKD1 genomic region and the estimated frequency of less than $4 \%$ of those in the ADPKD mutation database (http://pkdb.mayo.edu) may be an underestimate.

In individuals aged $<40$ years who are being considered as living related kidney donors, who have no cysts on renal ultrasound or five to ten cysts by MRI, genetic testing is a valuable additional tool to exclude ADPKD with certainty (Table 1). Although computed tomography scanning is used commonly in the evaluation of donor anatomy, including the renal vasculature and collecting system and is a sensitive test for the detection of renal cysts, it has not been validated as a screening tool to exclude ADPKD. In genetic testing, the recipient is first screened for PKD1 and PKD2 mutations and if a causal mutation is identified then focused mutation detection can be carried out on the prospective donor. If a pathogenic mutation in $P K D 1$ and $P K D 2$ is not identified in the recipient, a final decision on donation should be deferred at least until age 30 , when the absence of cysts by routine ultrasound has a NPV of $98.3 \%$. Genetic testing should also be offered before a final decision on donor suitability is made in at-risk individuals aged $<30$ years with five to ten cysts by MRI or if an MRI is unavailable or contraindicated, where an ultrasound is negative for cysts.

\section{Autosomal Dominant Interstitial Kidney Disease}

Mutations in at least four genes are implicated in autosomal dominant interstitial kidney disease: $M U C 1$ encoding mucin 1 (1q22 and OMIM 158340) causing medullary cystic kidney disease 1 (MCKD1) [26••], UMOD encoding uromodulin (16p12.3 and OMIM 191845) causing MCKD2 [27], HNF1B encoding hepatocyte nuclear factor-1 $\beta$ (17q12 and OMIM 189907) causing one of many forms of developmental renal disease or a form of maturity-onset diabetes or both and called RCAD syndrome [28, 29], and REN encoding renin (1q32.1 and OMIM 179820) [30]. MCKD1, MCKD2, and RCAD, which are more common, are discussed here.

\section{Uromodulin-Associated Kidney Disease (MCKD2)}

Mutations in the $U M O D$ gene are classically associated with clinical manifestations of hyperuricemia in childhood, gout beginning in teenage years, and slowly progressive CKD with the need for renal replacement therapy in the third through the seventh decades of life $[31,32 \bullet \cdot$. Disease resulting from mutations in the $U M O D$ gene has been called familial juvenile hyperuricemic nephropathy (FJHN), MCKD2, hereditary nephropathy with hyperuricemia and gout, uromodulin storage disease, and glomerulocystic kidney (GCK) disease depending on the clinical phenotype. A clinical distinction among these terms is not warranted because they represent the spectrum of the same disease [33]. Moreover, with the availability of genetic 
Table 1 Some examples of genetic renal diseases

\begin{tabular}{|c|c|c|c|}
\hline Disease & Affected gene & Gene product & Donation from a related donor? \\
\hline \multicolumn{4}{|l|}{ Autosomal-dominant disorders } \\
\hline ADPKD type 1 & $P K D 1$ & Polycystin 1 & Yes if disease excluded \\
\hline ADPKD type 2 & PKD 2 & Polycystin 2 & Yes if disease excluded \\
\hline Atypical HUS & $\begin{array}{l}C F H, C F B, C F I \\
\quad C 3, M C P, T H B D\end{array}$ & $\begin{array}{l}\text { Complement regulatory } \\
\text { proteins }\end{array}$ & No \\
\hline \multirow[t]{2}{*}{ Interstitial kidney disease } & $\begin{array}{l}\text { UMOD } \\
\text { MUC1 }\end{array}$ & $\begin{array}{l}\text { Uromodulin } \\
\text { Mucin } 1\end{array}$ & \multirow[t]{2}{*}{ Yes if disease excluded } \\
\hline & $H N F 1 B$ & $\begin{array}{l}\text { Hepatocyte nuclear } \\
\text { factor- } 1 \mathrm{~b}\end{array}$ & \\
\hline \multirow[t]{2}{*}{ FSGS/SRNS } & $\begin{array}{l}\text { ACTN4 } \\
\text { INF2 }\end{array}$ & $\begin{array}{l}\alpha \text {-actinin } 4 \\
\text { formin }\end{array}$ & \multirow[t]{2}{*}{ Yes if disease excluded } \\
\hline & TRPC6 & TRPC6 & \\
\hline \multicolumn{4}{|l|}{ Autosomal-recessive disorders } \\
\hline \multirow{3}{*}{$\begin{array}{l}\text { Congenital nephrotic syndrome } \\
\text { or autosomal-recessive SRNS }\end{array}$} & NPHS1 & Nephrin & Yes if heterozygous \\
\hline & NPHS 2 & Podocin & Caution with R229Q or R138Q \\
\hline & $P L C E$ & Phospholipase $\mathrm{C} \varepsilon$ & Yes if heterozygous \\
\hline Alport syndrome & COL $4 A 3, C O L 4 A 4$ & $\alpha 3$ or $\alpha 4$ type IV collagen & Yes if heterozygous \\
\hline TBMN & COL4A3, COL $4 A 4$ & $\alpha 3$ or $\alpha 4$ type IV collagen & Caution if heterozygous \\
\hline Nephronophthisis & NPHP1 to 18 & Various & Yes if heterozygous \\
\hline ARPKD & PKHD1 & Fibrocystin & Yes if heterozygous \\
\hline ApoL1-related disease & $A P O L 1$ & Apolipoprotein L1 & No if two copies $(\mathrm{G} 1, \mathrm{G} 1 ; \mathrm{G} 2 / \mathrm{G} 2$ or G1/G2) \\
\hline Primary hyperoxaluria & $\begin{array}{l}A G X T \\
G R H P R\end{array}$ & $\begin{array}{l}\text { alanine glycoxylate } \\
\text { aminotransferase } \\
\text { glyoxylate reductase/ } \\
\text { hydroxypyruvate } \\
\text { reductase }\end{array}$ & $\begin{array}{l}\text { Yes if heterozygous; (combined liver kidney preferred for } \\
\text { recipient) }\end{array}$ \\
\hline Atypical HUS & $D G K E$ & diacylglycerol kinase $\varepsilon$ & Unknown \\
\hline \multicolumn{4}{|l|}{ X-linked diseases } \\
\hline Alport syndrome & $C O L 4 A 5$ & $\alpha 5$ type IV collagen & $\begin{array}{l}\text { Yes in males and females if disease excluded; caution in older } \\
\text { heterozygous females if no or minimal phenotype }\end{array}$ \\
\hline Fabry disease & $G L A$ & $\alpha$-Galactosidase & $\begin{array}{l}\text { Yes in males and females if disease excluded; caution in older } \\
\text { heterozygous females if no or minimal phenotype }\end{array}$ \\
\hline
\end{tabular}

testing, it has become clear that neither medullary cysts nor juvenile gout are defining features of MCKD2. Rather, the features are non-specific and include general findings of a chronic tubulointerstitial disease such as small kidneys, minimal proteinuria, absent hematuria, and otherwise a bland urine. $U M O D$ mutation leads to an abnormal protein retention within the endoplasmic reticulum (ER) of the thick ascending limb of the loop of Henle (TALH), disrupting its function. The abnormal uromodulin protein is identified in renal tubular cells, and this dominant negative effect may be the primary mechanism for progressive chronic kidney disease (CKD) [34] .

\section{$M C K D 1$}

MCKD1 is characterized by features of a chronic tubulointerstitial disease with occasional cortical cysts on renal imaging, minimal proteinuria, bland urinary sediment, and no other associated features other than progressive CKD [35•]. Unlike uromodulin-associated disease, juvenile gout is not a typical or classic feature of this disease but has been rarely described. MCKD1 arises from a heterozygous mutation in $M U C 1$, where a single cytosine is inserted into an extremely long GC-rich region that encodes a variable number tandem repeat (VNTR), causing a frameshift mutation of mucin 1 . The mutant protein can be detected by immunohistochemistry in epithelial cells of the distal nephron and, like uromodulinassociated disease, MCKD1 may occur from disruption of epithelial cell function from the retained abnormal protein $[26 \bullet \cdot$. The disease appears to be highly penetrant although it can arise as a new mutation, and family history may be absent. 


\section{RCAD/MODY5}

The principal phenotypic manifestations seen with heterozygous mutations in $H N F 1 B$, a transcription factor important for genitourinary, pancreatic, and hepatic development are a variety of renal developmental disorders or a form of maturityonset diabetes of the young (MODY5) or both [36]. Renal cysts are the most commonly described renal abnormality, but HNF1B mutations can phenocopy FJHN and MCKD and hence this disorder is described under autosomal-dominant interstitial diseases. Other renal defects, including renal hypoplasia, multicystic renal dysplasia, unilateral renal agenesis, horseshoe kidney, and glomerulocystic kidney disease, can also be caused by mutations in HNF1B [37]. The combination of renal cysts and MODY5 has resulted in the name 'renal cysts and diabetes', although renal cysts are not the only renal manifestation and the renal defects and diabetes may not be present together. Other syndromic features of RCAD include genitourinary abnormalities such as hypospadias in males and vaginal aplasia in females, abnormal liver function tests, and hyperuricemia with early-onset gout.

RCAD may be one of the more common inherited kidney diseases. In a cohort of 160 European adult subjects with renal diseases of unknown etiology, a mutation or deletion in $H N F 1 B$ was identified in $24 \%$ of patients [38•]. In another European study of 377 subjects with a variety of kidney phenotypes, mutations in $H N F 1 B$ were identified in $20 \%$ of cases [39॰]. More than half the mutations were whole gene deletions and many of the deletions and mutations were novel and de novo.

\section{Testing of Transplant and Living Donor Candidates}

Transplant candidates with features of chronic tubulointerstitial disease (small kidneys, bland urine) and a positive family history may have mutations in $U M O D, M U C 1$, or HNF1B. UMOD and $H N F 1 B$ mutations may be associated with juvenile gout, while MUC1 mutations are not. Candidates with $H N F 1 B$ mutations may have a variety of renal developmental defects from vesicoureteric reflux, horseshoe kidneys, renal cysts, unilateral renal agenesis, or bilateral renal hypoplasia associated with or without maturity-onset diabetes of the young and/or pancreatic atrophy [36].

The genetics of these three diseases are similar to those of other autosomal-dominant diseases, resulting in many family members being affected, and a $50 \%$ probability that a child will inherit the abnormal allele from an affected parent. However, it is pertinent to note that $H N F 1 B$-associated disease has variable expressivity and is not very penetrant, while mutations in both $U M O D$ and $M U C 1$ are highly penetrant with age-dependent manifestations eventually presenting in all affected individuals. Both $H N F 1 B$ and MUC1 mutations can arise de novo, and family history may be lacking. If the genetic basis of disease in the transplant candidate is not already known, then consideration should be given to screening transplant candidates with possible inherited tubulointerstitial disease for mutations in $U M O D$, $M U C 1$, or $H N F 1 B$ and, if a pathogenic mutation is identified, then targeted mutation screening should be offered to the donor candidate. Since a majority of mutations in HNF1B are gene deletions, if standard exonic PCR and sequencing is negative, additional testing for large genomic rearrangements of $H N F 1 B$ by techniques such as multiplex ligation-dependent probe amplification (MLPA) may be necessary [38•]. Mutation screening for $U M O D, M U C 1$, and $H N F 1 B$, including MLPA, are commercially available. Donor candidates who have obvious urinary abnormalities by laboratory testing and/or imaging and have a $50 \%$ risk of having the disease or are confirmed to carry the mutant allele should not be kidney donors (Table 1).

3. The Inherited Podocytopathies (Congenital Nephrotic Syndrome/Steroid-Resistant Nephrotic Syndrome/Hereditary Focal Segmental Glomerulosclerosis)

The discovery of genes associated with congenital nephrotic syndrome (CNS), steroid-resistant nephrotic syndrome (SRNS), and hereditary focal segmental glomerulosclerosis (FSGS) has greatly enhanced the understanding of podocyte biology and pathogenesis of these inherited podocytopathies. Mutations in NPHS1 (nephrin), NPHS2 (podocin), PLCE1 (phospholipase C epsilon 1), MYOIE (mysoin 1E), ARHGDIA (arhgdia), LAMB2 (laminin $\beta$ 2), and $C U B N$ (cubulin) are characterized by an autosomal recessive pattern of inheritance with the onset of disease in utero, at birth, or in early childhood. CNS occurs within 3 months of age. The characteristic histology in the Finnish type is immature glomeruli, mesangial hypercellularity, and pseudocystic tubular dilatation [40]. In the non-Finnish type, the histology is either that of FSGS or diffuse mesangial sclerosis (DMS). Childhood-onset SRNS is usually autosomal recessive or sporadic, with the common histological correlate of FSGS and less often of DMS. Mutations in ACTN4 ( $\alpha$-actinin4), CD2AP (CD2-associated protein), TRPC6 (transient receptor potential cation channel, subfamily $\mathrm{C}$ member 6), INF2 (formin), $A R H G$ AP24 (RhoGAP), WT1 (Wilms tumor 1), and PAX2 cause autosomal-dominant FSGS, which is typically steroid resistant, and in most patients with dominant disease the onset is in late childhood or adulthood $[41 \bullet \bullet]$. The availability of genetic testing demonstrates that there is some overlap between genes that cause the three clinical entities of CNS, childhood-onset SRNS, and later-onset, usually autosomal-dominant, FSGS. Furthermore, $P A X 2$ mutations can also cause papillorenal syndrome, which is a developmental disorder that includes congenital anomalies of the kidney or urinary tract and retinal or optic nerve coloboma $[42,43]$. In addition, there are syndromic forms of SRNS, for example, Denys Drash and Frasier syndrome caused by $W T 1$ mutations and nail-patella syndrome caused by $L M X 1 B$ mutations. In patients with CNS/SRNS or FSGS, knowledge of the 
type of mutation may be useful to determine appropriate treatment strategies and to estimate the risk of recurrence after transplantation as well as for the evaluation of a related living donor $[41 \bullet \bullet, 44]$.

\section{Autosomal-Recessive (Steroid-Resistant) Nephrotic Syndrome}

CNS of the Finnish type is caused by autosomal-recessive mutations in NPHS1, with individuals presenting with massive proteinuria in utero and nephrotic syndrome within the first few weeks of life [45]. However, NPHS1 mutations can occasionally cause SRNS in childhood [46]. Homozygous or compound heterozygous mutations in NPHS2 are the most common genetic cause of SRNS in children [41••]. The clinical manifestations of NPHS2 mutations are variable, and patients can present with CNS or as SRNS in children or even FSGS in older children or young adults $[47,48]$. Simultaneous NPHS1 and NPHS2 mutations with a homozygous mutation in one gene and a heterozygous mutation in the other have also been described in CNS $[47,49]$. In a study of 152 patients with sporadic FSGS, $21 \%$ had homozygous or compound heterozygous NPHS2 mutations, indicating that these individuals had autosomal-recessive FSGS without a family history [50]. Adult-onset SRNS/FSGS has been described in patients heterozygous for a pathogenic NPHS2 mutation together with a variant of NPHS2 where Arg at position 229 is replaced by Gln (p.R229Q) [51]; this variant exhibits partial loss of function and is found in $3.6 \%$ of the normal population [52]. This raises the possibility that a donor related to a child who has two pathogenic NPHS2 mutations might carry the common p.R229Q variant together with one abnormal NPHS2 allele, putting that donor at risk of adult-onset SRNS or FSGS. Mutations in WT1 and PLCE1 classically cause DMS that can present rarely as congenital and more often as infantile or childhood SRNS [48]. Recessive mutations in MYO1E, $A R H G D I A$, and CUBN appear to be less common causes of recessive SRNS. FSGS in African-Americans may be related to homozygous or compound heterozygous inheritance of common APOL1 gene variants and are discussed later.

\section{Autosomal-Dominant (Steroid-Resistant) Nephrotic Syndrome}

Mutations in INF2 are the most common identified cause of later-onset dominant FSGS, accounting for about $12 \%$ of dominant FSGS; ACTN4 and TRPC6 account for about 2$6 \%$ [44]. Most patients with the autosomal-dominant nephrotic syndrome present as FSGS in adolescents or adulthood although occasionally the presentation occurs even in childhood. In a study to investigate the role of NPHS2 polymorphisms in sporadic late-onset FSGS, 377 FSGS cases and 919 controls were genotyped for NPHS2 [53]. Although no homozygous or compound heterozygous mutations were identified, both African American and European Americans who carry one copy of the common disease-causing mutation R138Q allele of NPHS2 appear to be at increased risk for developing FSGS. This implies that first-degree relatives of patients who have NPHS2-related recessive SRNS or FSGS may be at increased risk for SRNS or FSGS, even if they carry just one abnormal allele. Mutations in CD2AP, ARHGAP24, and PAX2 appear to be less common causes of dominant SRNS.

Testing of Transplant and Living Donor Candidates

There are reported examples of living donors with a family history of FSGS who developed nephrotic syndrome themselves after kidney donation [54]. Two donors who had apparently normal renal function and a negative urinalysis at the time of donation developed ESRD several years later, with at least one of them showing biopsy features compatible with FSGS. These observations, though rare, highlight the risk of later development of FSGS in living related donors and emphasize the importance of determining the genetic cause of nephrotic syndrome in the transplant candidate with a biologically related donor. Transplant candidates who receive kidneys from at-risk living donors may also be at heightened risk of FSGS in the transplanted kidney, and this may be one explanation for the apparent incidence of recurrent disease seen in inherited FSGS.

Infants and very young children presenting with CNS or familial SRNS should be screened for mutations in NPHSI and $N P H S 2$, as these are found in as many as $100 \%$ of CNS and $60 \%$ of infantile-onset SRNS [48]. More than 100 pathogenic mutations in NPHS2 have been described as causing the inherited nephrotic syndromes, and some increase the risk of FSGS/SRNS even when inherited as a single copy [53]. It is less clear whether adults with sporadic FSGS who present for transplantation with a living related donor should be screened for mutations in NPHS2, although testing for the R138Q allele and R229Q in young adults is relatively easy. In one study, heterozygosity for R138Q of NPHS2 was associated with a fivefold increased risk of sporadic FSGS but no increase in allele frequency for R229Q was seen; in another study, the R229Q variant was found at twice the allele frequency in sporadic FSGS than in controls, although this did not reach statistical significance $[52,53]$. Donors who carry at-risk heterozygous alleles such as R229Q or R138Q of NPHS2 should be counseled about the heightened risk of FSGS/SRNS (Table 1).

Children and young adults may have specific extra renal findings that suggest a form of syndromic SRNS and merit a more detailed phenotypic characterization, referral to clinical genetics service, and/or targeted genetic testing. For example, triangular or absent lunulae in fingernails or absent or hypoplastic patella should prompt a consideration of nail-patella syndrome; the presence of thrombocytopenia with giant 
platelets and sensorineural deafness should raise suspicion for Epstein or Fechtner syndrome.

Transplant candidates with later-onset autosomal-dominant FSGS should be considered for screening of INF2, ACTN4, and TRPC6 and possibly for the other genetic causes of dominant FSGS. A recent study that included familial and sporadic cases of adult-onset FSGS/SRNS found causal mutations in about $10 \%$ of cases $[55,56 \cdot \bullet]$. Identification of the genetic mutation in the transplant candidate could then be followed by targeted screening of the donor candidate. However, if not already known, a number of genes will need to be tested in the transplant candidate in later-onset SRNS/FSGS, and this may not be practical until targeted exome sequencing platforms that contain a panel of nephrotic syndrome genes become commonly available. Donors who carry the same mutant allele as identified in the transplant candidate with autosomal-dominant FSGS should be excluded from donation (Table 1).

\section{APOL1-Related Disease}

Recently, APOL1 gene variants G1 and G2 were identified at high frequency in African-Americans with a variety of nondiabetic kidney diseases, including HIV-associated nephropathy, FSGS, hypertension-related nephropathy, sickle cell nephropathy, and lupus kidney disease [57]. The currently accepted model is that homozygosity or compound heterozygosity for the G1 and G2 alleles causes autosomal-recessive predisposition to progressive CKD in patients, with the risk for kidney disease accentuated in the presence of hypertension, HIV, lupus, or sickle cell disease $[58 \bullet \bullet, 59,60 \bullet, 61]$. FSGS, HIV-associated nephropathy, arteriolar nephrosclerosis, and hypertension attributed ESRD appear to be members of a single disease spectrum [9•]. The carrier state for either of two coding variants $(\mathrm{G} 1, \mathrm{G} 2)$ is protective against the parasite Trypanosoma brucei rhodesiense that causes sleeping sickness endemic to sub-Saharan Africa, and this heterozygous advantage appears to have driven natural selection, and explains why one copy of G1 or G2 is present in approximately $33-62 \%$ of West Africans and about $37 \%$ of African-Americans, whereas the variants are virtually absent in individuals of European, Native American, and East Asian descent and are very rare in some parts of North Africa [57, $58 \bullet^{\circ}$ ]. APOL1-related renal disease should be considered in transplant candidates of Sub-Saharan African descent with FSGS, HIV-associated nephropathy, and hypertensive nephrosclerosis and when ESRD occurs in the context of a family history of CKD or ESRD [62••].

\section{Testing of Living Donor Candidates}

APOL1 risk variants were genotyped in 106 AfricanAmerican deceased kidney donors at one center, and allograft survival was assessed in the 136 resulting transplants. Kidneys from African-American deceased donors that harbored two APOL1 risk variants failed far more rapidly after transplantation than those with zero or one risk variant [63]. More important to the focus of this report, there is a higher risk of ESRD in African-American living donors [2, 5••, 64]. As African-Americans tend to donate at a younger average age and are more likely related to their recipient, the demographic differences in long-term post-donation risks for AfricanAmericans may be related specifically to the genetic variants of APOL1. The probability that an African-American in the general population carries two risk alleles is $12 \%$ and increases to about $72 \%$ in an African-American with FSGS. The offspring of two individuals, one without kidney disease and one with FSGS, has a $28 \%$ risk of carrying two risk alleles and a seven- to tenfold higher risk of developing FSGS or hypertensive CKD, even without donation. While more data and follow-up are needed to evaluate the use of APOL1 gene analysis in the risk stratification and selection of potential living donors, it seems prudent, until more data are available, to genotype biologically related African-American living donors whose recipient has FSGS, hypertensive kidney disease, or HIV nephropathy. If two risk alleles for G1 and/or G2 are found, then the donor candidate should be counseled about the substantially higher risk of kidney disease and advised against donation [65].

\section{Atypical Hemolytic Uremic Syndrome}

Atypical hemolytic uremic syndrome (aHUS) is a relatively rare syndrome of renal failure, hemolysis, and thrombocytopenia and is now accepted as a disorder that arises for the most part from excessive activation of the alternate complement pathway on endothelial cells [66]. Although the classic presentation is with an acute thrombotic microangiopathy on renal biopsy, with microangiopathic hemolysis evident in peripheral blood together with a low complement C3 level, some patients with aHUS present with ESRD or advanced CKD with or without heavy proteinuria, where histology may be absent or atypical and with subtle or no evidence of hemolysis or thrombocytopenia. Complicating the picture, aHUS cannot always be reliably differentiated from other thrombotic microangiopathies such as thrombotic thrombocytopenic purpura (TTP), lupus vasculitis, antiphospholipid antibody syndrome, malignant hypertension, and scleroderma renal crisis [67••]. Although mutations in complement regulatory genes such as complement factor $\mathrm{H}(\mathrm{CFH})$, membrane cofactor protein $(M C P)$, factor I $(C F I)$, factor B $(C F B)$, and complement $C 3$ were initially identified in aHUS, the list of genes associated with aHUS has grown to include proteins in the coagulation pathway such as thrombomodulin (THBD), plasminogen (PLG), and factor 12 (F12) [68, 69]. Inheritance of an abnormal allele to any one of these proteins confers 
susceptibility to aHUS, and an environmental trigger such as pregnancy, infection, surgery, or drugs appears to be necessary for the disease to manifest, which may explain the penetrance of about $50 \%$. Autosomal-recessive mutations in a podocyte protein diacylglycerol kinase $\varepsilon$ (DGKE), where complement may not be involved, typically present with aHUS by 1 year of age $[70,71]$. Some cases of aHUS are secondary to CFH autoantibodies, which are more frequently seen in patients with homozygous deletion of two genes, CFHR 1 and CFHR 3 [72]. No mutations or antibodies are identified in about 20$30 \%$ of sporadic cases of aHUS, suggesting the existence of other genes that cause or predispose to aHUS. Prior to the advent of eculizumab, the monoclonal antibody directed to complement $\mathrm{C} 5$, there was a high rate of progression to ESRD and a high rate of disease recurrence post-transplantation $[66$, 73•].

\section{Testing of Transplant and Living Donor Candidates}

A complete genetic workup and an assessment for $\mathrm{CFH}$ autoantibodies should be considered for transplant candidates with aHUS, and eculizumab should be used peri-operatively and post-operatively to prevent relapses of aHUS post-transplantation. Since $M C P$ and $D G K E$ are kidney-expressed proteins, post-transplant disease recurrence is not expected; however, about $10 \%$ of patients have mutations in multiple genes and $20-30 \%$ have no clearly identified mutations and thus some patients with $M C P$ and/or DGKE mutations are likely to have an unrecognized mutation elsewhere. Furthermore, although DGKE mutations are not predicted to affect complement activation, some patients with $D G K E$-related aHUS respond to eculizumab [70].

A living donor transplant is preferable for transplant candidates with aHUS, especially if they are not already established on eculizumab so that pre-operative eculizumab and perhaps plasma therapy can be planned and administered [73•]. A large and increasing list of candidate genes that confer susceptibility to aHUS makes genetic screening of a related living donor less practical than many other diseases with limited locus heterogeneity, until targeted multigene sequencing platforms are available. A recently described in vitro test that detects abnormal C5b-9 deposition on activated endothelial cells in apparently healthy people who carry the risk allele is promising, but its performance as a screening tool for related living donors is unknown [74]. Given that as many as $30 \%$ of aHUS patients do not have an identifiable genetic mutation, and others have multiple affected genes, a negative genetic screen cannot eliminate the risk for aHUS in a screened related living donor. Based on the known genetic risk of aHUS and the false-negative, rate it is prudent to discourage at-risk candidates from donating (Table 1).
6. Alport Syndrome and Thin Basement Membrane Nephropathy

Alport syndrome is characterized by a lamellated glomerular basement membrane (GBM) with an abnormal collagen IV composition $[75,76]$ secondary to mutations in the COL4A3, COL4A4, or COL4A5 genes [77, 78], which encode the alpha 3,4 , and 5 chains of type IV collagen, respectively. Alport syndrome is characterized by renal failure, sensorineural deafness, and ocular abnormalities such as lenticonus and perimacular pigmentary flecks. Some $85 \%$ of families have $\mathrm{X}$ - linked dominant inheritance with mutations in COL4A5 [79], and most of the others have autosomal-recessive disease with homozygous or compound heterozygous mutations in both copies of COL4A3 or COL4A4 [78]. Autosomaldominant inheritance is very rare and results from heterozygous COL4A3 or COL4A4 mutations [80]. Thin basement membrane nephropathy (TBMN) can be caused by heterozygous COL $4 A 3$ or COL $4 A 4$ mutations and often represents the 'carrier' state of autosomal-recessive Alport syndrome [81]. The diagnosis of Alport syndrome is confirmed by the presence of a lamellated or thin GBM on renal biopsy and the presence of sensorineural hearing loss together with a family history or by demonstration of a pathogenic mutation in the COL4A5 gene or two pathogenic COL $4 A 3$ or COL4A4 mutations. In hemizygous males with $\mathrm{X}$-linked disease and in homozygous recessive Alport syndrome of either gender, there is expected to be absent staining for the alpha 3 , alpha 4 , and alpha 5 chains of type 4 collagen in the GBM. The disease is highly penetrant in hemizygous males with renal failure usually before the age of 30 years. About $93 \%$ of heterozygous females with the X-linked Alport syndrome have hematuria and $15 \%$ of heterozygous females develop ESRD by the age of 60 years in large cohort studies [82]. All affected members of a family with X-linked Alport syndrome, including females, should be identified because of their own risk, and their children's risk of renal failure.

\section{Testing of Transplant and Living Donor Candidates}

For any female with X-linked Alport syndrome, each of her sons has a $50 \%$ risk of having Alport syndrome and developing renal failure, and each of her daughters has a $50 \%$ risk of being affected in some fashion. In contrast, a male with Xlinked disease cannot transmit to his sons but all of his daughters will be 'carriers' and almost all eventually have detectable disease $[82,83]$. Most mothers of an affected male with X-linked Alport syndrome are heterozygous for the mutant allele and will likely have clinical manifestations; however, about $15 \%$ of males with X-linked Alport syndrome are affected as the result of a spontaneous gene mutation, and their mothers are not carriers. Genetic analysis is recommended for women at risk (female siblings of an affected male) 
without clinical manifestations, and clearly heterozygous females (mothers or daughters of affected male) should be strongly discouraged from kidney donation because of their own increased risk of future renal impairment and hypertension, unless they are older, with a minimal phenotype [84].

With recessive inheritance, disease typically occurs in a single generation, and males and females are affected in equal proportion and with equal severity. Recessive inheritance is also suspected when a young female has renal failure, hearing loss, and ocular abnormalities and when the father of an affected male has hematuria, but this must be confirmed with genetic testing. When the mutation in any family member is known, genetic testing can be used to confirm the affected status. Donor candidates with only one of the mutations that contribute to autosomal-recessive Alport syndrome (parents, offspring, and some siblings) may have TBMN with hematuria as the phenotype. It is important to remember that autosomal-dominant Alport syndrome arises from heterozygous mutations in COL4A3 or COL4A4 and, unless the mutation in one allele of COL4A3 or COL4A4 is known to segregate with TBMN in a given pedigree, one cannot be certain that heterozygous mutations of COL4A3 or COL4A4 will be limited to a TBMN phenotype [85]. Further, although the prognosis for TBMN was once considered to be excellent, there is a reported increased risk of proteinuria and renal impairment, which in one large series from Cyprus was seen in $26 \%$ of people with microscopic hematuria $[81,86]$. Successful deceased donor renal transplants from donors with TBMN have been reported [81, 87, 88]. Nevertheless, there is a risk of serious kidney disease in some living donors with familial hematuria and TBMN on biopsy, and genetic testing should be considered, and related donor candidates with microscopic hematuria, TBMN, or a positive genetic screen should be discouraged from donation (Table 1).

\section{Fabry Disease}

Fabry disease is an X-linked genetic disorder of glycosphingolipid (GL) metabolism resulting from deficient activity of the lysosomal enzyme $\alpha$-galactosidase $\mathrm{A}(\alpha$-Gal A) that results in the accumulation of the neutral GL globotriaosylceramide $(\mathrm{Gb} 3)$ in the walls of small blood vessels, nerves, cardiomyocytes, and renal glomerular and tubular epithelial cells. As expected for an X-linked disorder, severe clinical manifestations occur in hemizygous males, whereas heterozygous females exhibit a variable but typically less severe course. However, heterozygous female individuals may be affected similarly to hemizygous males and have advanced systemic disease due to random X-chromosomal inactivation [89]. A recent analysis of the Fabry disease registry showed that $14 \%$ of male and $2 \%$ of female patients had a history of ESRD [90]. Fabry disease is confirmed by biochemical and genetic testing. Deficiency of $\alpha$-gal A, most commonly measured in whole blood or leukocytes, and identification of a disease-causing mutation in the GLA gene located on Xq22.1 confirms the diagnosis. Measurement of $\alpha$-gal A enzyme activity alone is not reliable for diagnosis of Fabry disease in females because obligate heterozygotes have variable levels of $\alpha$-gal A that can overlap with enzyme levels found in healthy controls. A slit lamp examination of the eyes can detect early disease in heterozygous females, although the sensitivity and NPV of this test in females has not been established. In females, the only definite confirmation of Fabry disease is by identification of a pathogenic mutation in the GLA gene.

\section{Testing of Transplant and Living Donor Candidates}

Fabry disease may be under-diagnosed in the ESRD population and should be considered in a transplant candidate who has a history of unexplained neuropathic pains, fevers, and reduced sweating or one who has peri-umbilical or scrotal angiokeratoma. If the transplant candidate is known to have Fabry disease, all donor candidates at $50 \%$ or greater risk of disease, which includes siblings, mothers of affected children, fathers of affected daughters, and all children of an affected mother, should be screened for Fabry's disease. All daughters of an affected father are at $100 \%$ risk and are not suitable living kidney donors (Table 1).

Those affected with Fabry disease may give the classic history of acroparesthesias and hypohidrosis although these, like the findings of angiokeratomata on skin exam does not have adequate sensitivity to diagnose the disease in heterozygous females. All males at risk should, at a minimum, have a negative urinalysis and a normal leucocyte $\alpha$-Gal A. A slit lamp exam with a normal leucocyte $\alpha$-Gal A or genetic sequencing of GLA is necessary in at-risk women. Targeted screening for familial mutation in GLA has an NPV of $100 \%$ and is superior to a normal leucocyte $\alpha$-Gal A and a normal slit lamp exam.

\section{Challenges in Genetic Diagnosis}

There are a number of challenges in the evaluation of a living donor who may be at increased genetic risk for serious kidney disease. First, some type of genetic diseases may appear to be sporadic because of limited penetrance or variable expressivity, and it may not be immediately apparent that the related recipient candidate has a genetic kidney disease. Second, many patients with ESRD do not have a clearly established etiology for their kidney disease yet are often diagnosed to have diabetic or hypertensive or other kidney disease with inadequate evidence to support that label. Third, although a few genetic diseases, such as ADPKD, can be diagnosed in 
the vast majority of patients on the basis of clinical, laboratory, and imaging studies, many other monogenic diseases require confirmation by genetic testing. Fourth, some diseases exhibit locus heterogeneity, that is, the disease can be caused by mutations in multiple genes (e.g. juvenile nephrophthisis, autosomal-dominant FSGS), which would require systematic screening of multiple genes, which may be expensive and time consuming. Finally, the technology for identifying genetic mutations is rapidly evolving, and clinicians may not be aware of the best options for genetic testing or be comfortable in interpreting equivocal results or 'incidental' findings.

\section{Conclusions}

We have discussed approaches to screen for and exclude a few genetic diseases in a donor candidate. It is not possible to discuss every known genetic form of progressive CKD, and, when in doubt, the transplant center should seek consultations with nephrologists with genetic expertise or with a clinical genetic service.

Living donation to biologically related individuals with kidney disease requires careful evaluation, including genetic testing where indicated, to minimize harm to a living donor who may be at risk but not manifest signs of disease. There are a large number of monogenic renal diseases, from the common and easily diagnosed ADPKD to the rare and more difficult to recognize diseases that, nevertheless, pose a serious risk to a person who harbors the genetic mutation and wants to offer one of their kidneys to help another. It is the solemn responsibility of the transplant community to exercise due diligence in evaluating the genetic risk of future kidney disease in every living kidney donor and to counsel and, where appropriate, caution the potential living donor about the risks of genetic renal disease and the unknown, possibly deleterious, additive effects of nephrectomy in those at risk.

Some donors related to an individual with an autosomalrecessive disease (e.g. primary hyperoxaluria, juvenile nephronophthisis, Bardet-Biedl syndrome, congenital nephrotic syndrome) will carry one disease allele. While the heterozygous carrier state is generally not considered to pose a significant renal risk, the risk of nephrectomy in individuals with some heterozygous variants (e.g. R138Q or R229Q of NPHS2) is unknown. On the other hand, for diseases with agedependent penetrance, the residual lifetime risk of serious kidney disease diminishes with increasing age in donors who may carry one risk variant with no phenotype at presentation and can be considered when counseling donors about the risks of nephrectomy.

Heterozygous 'carriers' of certain X-linked diseases (e.g. Alport's, Fabry's) may already have serious kidney disease at evaluation while others without manifest disease may nevertheless be at risk for progressive CKD. However, an older woman with the heterozygous mutation and minimal or no phenotype and otherwise normal renal function could be considered as a donor if the candidate has been counseled that the risk of disease is diminished but not eliminated.

For diseases with locus heterogeneity and multiple modes of inheritance (e.g. familial FSGS), extensive genetic screening introduces delays in donor evaluation and may not be considered by some to be practical. In other situations where the transplant candidate presents with apparent sporadic disease, screening for causal mutations in some genes may have a low yield, limiting clinical enthusiasm. In yet other situations, the phenotype may not be precise enough and without suspicion for a specific disease or syndrome, the appropriate genetic test cannot be requested. In the future, it should be possible to quickly and economically screen a living donor for a large number of diseases simultaneously with technologies like targeted exome sequencing in a relatively unbiased fashion and to improve the risk estimates that we can provide to potential living donors.

\section{Compliance with Ethics Guidelines}

Conflict of Interest S. Kuppachi, R.J.H Smith, and C.P. Thomas declare that they have no conflict of interest.

Human and Animal Rights and Informed Consent This article does not contain any studies with human or animal subjects performed by any of the authors.

\section{References}

Papers of particular interest, published recently, have been highlighted as:

- Of importance

•• Of major importance

1. Ibrahim HN, Foley R, Tan L, Rogers T, Bailey RF, et al. Long-term consequences of kidney donation. N Engl J Med. 2009;360:459 69.

2. Lentine KL, Schnitzler MA, Xiao H, Saab G, Salvalaggio PR, et al. Racial variation in medical outcomes among living kidney donors. N Engl J Med. 2010;363:724-32.

3.• Cherikh WS, Young CJ, Kramer BF, Taranto SE, Randall HB, et al. Ethnic and gender related differences in the risk of end-stage renal disease after living kidney donation. Am J Transplant Off J Am Soc of Transplant Am Soc Transplant Surg. 2011;11:1650-5.

4.• Mjoen G, Hallan S, Hartmann A, Foss A, Midtvedt K, et al. Longterm risks for kidney donors. Kidney Int. 2014;86:162-7.

5.• Muzaale AD, Massie AB, Wang MC, Montgomery RA, McBride $\mathrm{MA}$, et al. Risk of end-stage renal disease following live kidney donation. JAMA J Am Med Assoc. 2014;311:579-86. The above four papers are well-conducted studies on large cohorts examining the risk from living kidney donation.

6. Matas AJ, Smith JM, Skeans MA, Thompson B, Gustafson SK, et al. OPTN/SRTR 2012 annual data report: kidney. Am J Transplant. 2014;14:11-44. 
7. Sharma SG, Bomback AS, Radhakrishnan J, Herlitz LC, Stokes $\mathrm{MB}$, et al. The modern spectrum of renal biopsy findings in patients with diabetes. Clin J Am Soc Nephro: CJASN. 2013;8:1718-24.

8. Christensen PK, Larsen S, Horn T, Olsen S, Parving HH. Causes of albuminuria in patients with type 2 diabetes without diabetic retinopathy. Kidney Int. 2000;58:1719-31.

9. Bostrom MA, Freedman BI. The spectrum of MYH9-associated nephropathy. Clin J Am Soc Nephro: CJASN. 2010;5:1107-13. This paper discusses the relationship between genetic disorders and the high incidence of ESRD in African American.

10. Rossetti S, Strmecki L, Gamble V, Burton S, Sneddon V, et al. Mutation analysis of the entire PKD1 gene: genetic and diagnostic implications. Am J Hum Genet. 2001;68:46-63.

11. Edghill EL, Stals K, Oram RA, Shepherd MH, Hattersley AT, et al. HNF1B deletions in patients with young-onset diabetes but no known renal disease. Diabet Med J Br Diabet Assoc. 2013;30: 114-7.

12. Biesecker LG, Green RC. Diagnostic clinical genome and exome sequencing. N Engl J Med. 2014;370:2418-25. A review that examines the diagnostic indications for clinical genome and exome sequencing and how to interpret results.

13. Koboldt DC, Steinberg KM, Larson DE, Wilson RK, Mardis ER. The next-generation sequencing revolution and its impact on genomics. Cell. 2013;155:27-38.

14. Trujillano D, Bullich G, Ossowski S, Ballarin J, Torra R, et al. Diagnosis of autosomal dominant polycystic kidney disease using efficient PKD1 and PKD2 targeted next-generation sequencing. Mol Genet Fenomic Med. 2014;2:412-21.

15. Kammermeier J, Drury S, James CT, Dziubak R, Ocaka L, et al. Targeted gene panel sequencing in children with very early onset inflammatory bowel disease-evaluation and prospective analysis. J Med Genet. 2014.

16. Renkema KY, Stokman MF, Giles RH, Knoers NV. Nextgeneration sequencing for research and diagnostics in kidney disease. Nat Rev Nephrol. 2014;10:433-44.

17. Iglesias CG, Torres VE, Offord KP, Holley KE, Beard CM, et al. Epidemiology of adult polycystic kidney disease, Olmsted County, Minnesota: 1935-1980. Am J Kidney Dis Off J National Kidney Foundation. 1983;2:630-9.

18. Wilson PD. Polycystic kidney disease. N Engl J Med. 2004;350: 151-64.

19.• Rossetti S, Consugar MB, Chapman AB, Torres VE, GuayWoodford LM, et al. Comprehensive molecular diagnostics in autosomal dominant polycystic kidney disease. J Am Soc Nephrol JASN. 2007;18:2143-60. This study evaluated molecular analysis for the diagnosis of ADPKD and showed the importance of molecular diagnostics in ADPKD.

20. Dicks E, Ravani P, Langman D, Davidson WS, Pei Y, et al. Incident renal events and risk factors in autosomal dominant polycystic kidney disease: a population and family-based cohort followed for 22 years. Clin J Am Soc Nephro: CJASN. 2006;1:710-7.

21. Hateboer N, v Dijk MA, Bogdanova N, Coto E, Saggar-Malik AK, et al. Comparison of phenotypes of polycystic kidney disease types 1 and 2. European PKD1-PKD2 Study Group. Lancet. 1999;353: $103-7$.

22. Ravine D, Gibson RN, Walker RG, Sheffield LJ, Kincaid-Smith P, et al. Evaluation of ultrasonographic diagnostic criteria for autosomal dominant polycystic kidney disease 1. Lancet. 1994;343:8247.

23. Pei Y, Obaji J, Dupuis A, Paterson AD, Magistroni R, et al. Unified criteria for ultrasonographic diagnosis of ADPKD. J Am Soc Nephrol JASN. 2009;20:205-12.

24.• Pei Y, Hwang YH, Conklin J, Sundsbak JL, Heyer CM, et al. Imaging-based diagnosis of autosomal dominant polycystic kidney disease. J Am Soc Nephrol: JASN. 2014. This is a recent paper that compares MRI and high-resolution ultrasound in the non-invasive diagnosis and screening of $A D P K D$.

25. Audrezet MP, Cornec-Le Gall E, Chen JM, Redon S, Quere I, et al. Autosomal dominant polycystic kidney disease: comprehensive mutation analysis of PKD1 and PKD2 in 700 unrelated patients. Hum Mutat. 2012;33:1239-50.

26.• Kirby A, Gnirke A, Jaffe DB, Baresova V, Pochet N, et al. Mutations causing medullary cystic kidney disease type 1 lie in a large VNTR in MUC1 missed by massively parallel sequencing. Nat Genet. 2013;45:299-303. This paper reports on a novel mechanism of disease in a complex region of the genome that could not be identified by NGS strategies.

27. Hart TC, Gorry MC, Hart PS, Woodard AS, Shihabi Z, et al. Mutations of the UMOD gene are responsible for medullary cystic kidney disease 2 and familial juvenile hyperuricaemic nephropathy. J Med Genet. 2002;39:882-92.

28. Lindner TH, Njolstad PR, Horikawa Y, Bostad L, Bell GI, et al. A novel syndrome of diabetes mellitus, renal dysfunction and genital malformation associated with a partial deletion of the pseudo-POU domain of hepatocyte nuclear factor-1beta. Hum Mol Genet. 1999;8:2001-8.

29. Bingham C, Ellard S, Allen L, Bulman M, Shepherd M, et al. Abnormal nephron development associated with a frameshift mutation in the transcription factor hepatocyte nuclear factor-1 beta. Kidney Int. 2000;57:898-907.

30. Zivna M, Hulkova H, Matignon M, Hodanova K, Vylet'al P, et al. Dominant renin gene mutations associated with early-onset hyperuricemia, anemia, and chronic kidney failure. Am J Hum Genet. 2009;85:204-13.

31. Moskowitz JL, Piret SE, Lhotta K, Kitzler TM, Tashman AP, et al. Association between genotype and phenotype in uromodulinassociated kidney disease. Clin J Am Soc Nephro: CJASN. 2013;8:1349-57.

32.• Bleyer AJ, Zivna M, Kmoch S. Uromodulin-associated kidney disease. Nephron Clin Pra. 2011;118:c31-6. An extensive review on uromodulin-associated kidney disease.

33. Scolari F, Caridi G, Rampoldi L, Tardanico R, Izzi C, et al. Uromodulin storage diseases: clinical aspects and mechanisms. Am J Kidney Dis Off J National Kidney Foundation. 2004;44: 987-99.

34. Bleyer AJ, Hart TC, Shihabi Z, Robins V, Hoyer JR. Mutations in the uromodulin gene decrease urinary excretion of Tamm-Horsfall protein. Kidney Int. 2004;66:974-7.

35. Bleyer AJ, Hart PS, Kmoch S. Hereditary interstitial kidney disease. Semin Nephrol. 2010;30:366-73. This is a comprehensive review on autosomal-dominant interstitial kidney disease.

36. Bingham C, Hattersley AT. Renal cysts and diabetes syndrome resulting from mutations in hepatocyte nuclear factor-1beta. Nephrol Dial Transplant Off Publ Eur Dial Transplant Assoc Eur Ren Assoc. 2004;19:2703-8.

37. Thomas CP, Erlandson JC, Edghill EL, Hattersley AT, Stolpen AH. A genetic syndrome of chronic renal failure with multiple renal cysts and early onset diabetes. Kidney Int. 2008;74:1094-9.

38. Edghill EL, Oram RA, Owens M, Stals KL, Harries LW, et al. Hepatocyte nuclear factor-1beta gene deletions-a common cause of renal disease. Nephrol Dial Transplant Off Publ Eur Dial Transplant Assoc - Eur Ren Assoc. 2008;23:627-35.

39. Heidet L, Decramer S, Pawtowski A, Moriniere V, Bandin F, et al. Spectrum of HNF1B mutations in a large cohort of patients who harbor renal diseases. Clin J Am Soc Nephro: CJASN. 2010;5: 1079-90. These two papers describe a high incidence of HNF1B mutations in patients with unknown renal disease.

40. Huttunen NP, Rapola J, Vilska J, Hallman N. Renal pathology in congenital nephrotic syndrome of Finnish type: a quantitative light microscopic study on 50 patients. Int J Pediatr Nephrol. 1980;1:10 6. 
41.• Rood IM, Deegens JK, Wetzels JF. Genetic causes of focal segmental glomerulosclerosis: implications for clinical practice. Nephrol Dial Transplant Publ Eur Dial Transplant Assoc Eur Ren Assoc. 2012;27:882-90. A review on inheritable genetic forms of FSGS and the clinical approach to treating those patients.

42. Eccles MR, Schimmenti LA. Renal-coloboma syndrome: a multisystem developmental disorder caused by PAX2 mutations. Clin Genet. 1999;56:1-9.

43. Barua M, Stellacci E, Stella L, Weins A, Genovese G, et al. Mutations in PAX2 associate with Adult-Onset FSGS. J Am Soc Nephrol JASN. 2014;25:1942-53.

44. Brown EJ, Pollak MR, Barua M. Genetic testing for nephrotic syndrome and FSGS in the era of next-generation sequencing. Kidney Int. 2014;85:1030-8.

45. Kestila M, Lenkkeri U, Mannikko M, Lamerdin J, McCready P, et al. Positionally cloned gene for a novel glomerular proteinnephrin-is mutated in congenital nephrotic syndrome. Mol Cell. 1998;1:575-82.

46. Philippe A, Nevo F, Esquivel EL, Reklaityte D, Gribouval O, et al. Nephrin mutations can cause childhood-onset steroid-resistant nephrotic syndrome. J Am Soc Nephrol JASN. 2008;19:1871-8.

47. Koziell A, Grech V, Hussain S, Lee G, Lenkkeri U, et al. Genotype/ phenotype correlations of NPHS1 and NPHS2 mutations in nephrotic syndrome advocate a functional inter-relationship in glomerular filtration. Hum Mol Genet. 2002;11:379-88.

48. Benoit G, Machuca E, Antignac C. Hereditary nephrotic syndrome: a systematic approach for genetic testing and a review of associated podocyte gene mutations. Pediatr Nephrol. 2010;25:1621-32.

49. Weber S, Gribouval O, Esquivel EL, Moriniere V, Tete MJ, et al. NPHS2 mutation analysis shows genetic heterogeneity of steroidresistant nephrotic syndrome and low post-transplant recurrence. Kidney Int. 2004;66:571-9.

50. Ruf RG, Lichtenberger A, Karle SM, Haas JP, Anacleto FE, et al. Patients with mutations in NPHS2 (podocin) do not respond to standard steroid treatment of nephrotic syndrome. J Am Soc Nephrol JASN. 2004;15:722-32.

51. Machuca E, Hummel A, Nevo F, Dantal J, Martinez F, et al. Clinical and epidemiological assessment of steroid-resistant nephrotic syndrome associated with the NPHS2 R229Q variant. Kidney Int. 2009;75:727-35.

52. Tsukaguchi H, Sudhakar A, Le TC, Nguyen T, Yao J, et al. NPHS2 mutations in late-onset focal segmental glomerulosclerosis: R229Q is a common disease-associated allele. J Clin Invest. 2002;110: 1659-66.

53. McKenzie LM, Hendrickson SL, Briggs WA, Dart RA, Korbet SM, et al. NPHS2 variation in sporadic focal segmental glomerulosclerosis. J Am Soc Nephrol JASN. 2007;18:2987-95.

54. Winn MP, Alkhunaizi AM, Bennett WM, Garber RL, Howell DN, et al. Focal segmental glomerulosclerosis: a need for caution in liverelated renal transplantation. Am J Kidney Dis Off J National Kidney Foundation. 1999;33:970-4.

55. Santin S, Bullich G, Tazon-Vega B, Garcia-Maset R, Gimenez I, et al. Clinical utility of genetic testing in children and adults with steroid-resistant nephrotic syndrome. Clin J Am Soc Nephro: CJASN. 2011;6:1139-48

56.• Joshi S, Andersen R, Jespersen B, Rittig S. Genetics of steroidresistant nephrotic syndrome: a review of mutation spectrum and suggested approach for genetic testing. Acta Paediatr. 2013;102: 844-56. This paper provides a good overview of genes associated with steroid-resistant nephrotic syndrome.

57. Limou S, Nelson GW, Kopp JB, Winkler CA. APOL1 kidney risk alleles: population genetics and disease associations. Adv Chronic Kidney Dis. 2014;21:426-33.

58.• Genovese G, Friedman DJ, Ross MD, Lecordier L, Uzureau P, et al. Association of trypanolytic ApoL1 variants with kidney disease in African Americans. Science. 2010;329:841-5. This paper describes the association between APOL1 variants and renal disease in African-Americans.

59. Freedman BI, Langefeld CD, Andringa KK, Croker JA, Williams $\mathrm{AH}$, et al. End-stage renal disease in African Americans with lupus nephritis is associated with APOL1. Arthritis Rheumatol. 2014;66: 390-6.

60.• Lipkowitz MS, Freedman BI, Langefeld CD, Comeau ME, Bowden DW, et al. Apolipoprotein L1 gene variants associate with hypertension-attributed nephropathy and the rate of kidney function decline in African Americans. Kidney Int. 2013;83:114-20. This study descibes the progression of kidney disease in the AASK cohort despite achieving good blood pressure targets and the strong association with APOL1 renal risk variants.

61. Ashley-Koch AE, Okocha EC, Garrett ME, Soldano K, De Castro LM, et al. MYH9 and APOL1 are both associated with sickle cell disease nephropathy. Br J Haematol. 2011;155:386-94.

62.• Pollak MR. Familial FSGS. Adv Chronic Kidney Dis. 2014;21: $422-5$. This is a recently published review on familial FSGS that is informative and extensive.

63. Reeves-Daniel AM, DePalma JA, Bleyer AJ, Rocco MV, Murea M, et al. The APOL1 gene and allograft survival after kidney transplantation. Am J Transplant Off J Am Soc Transplant and the Am Soc Transplant Surg. 2011;11:1025-30.

64. Gibney EM, King AL, Maluf DG, Garg AX, Parikh CR. Living kidney donors requiring transplantation: focus on African Americans. Transplantation. 2007;84:647-9.

65. Cohen DM, Mittalhenkle A, Scott DL, Young CJ, Norman DJ. African American living-kidney donors should be screened for APOL1 risk alleles. Transplantation. 2011;92:722-5.

66. Mele C, Remuzzi G, Noris M. Hemolytic uremic syndrome. Semin Immunopathol. 2014;36:399-420.

$67 . \bullet$ George JN, Nester CM. Syndromes of thrombotic microangiopathy. N Engl J Med. 2014;371:654-66. This is a recently published, extensive review on thrombotic microangiopathies, including aHUS.

68. de Cordoba SR, Hidalgo MS, Pinto S, Tortajada A. Genetics of atypical hemolytic uremic syndrome (aHUS). Semin Thromb Hemost. 2014;40:422-30.

69. Bu FX, Maga T, Meyer NC, Wang K, Thomas CP, et al. Comprehensive genetic analysis of complement and coagulation genes in atypical hemolytic uremic syndrome. J Am Soc Nephrol. 2014; $25: 55-64$

70. Westland R, Bodria M, Carrea A, Lata S, Scolari F, et al. Phenotypic expansion of DGKE-associated diseases. J Am Soc Nephrol. 2014;25:1408-14.

71. Lemaire M, Fremeaux-Bacchi V, Schaefer F, Choi MR, Tang WH, et al. Recessive mutations in DGKE cause atypical hemolyticuremic syndrome. Nat Genet. 2013;45:531-U102.

72. Hofer J, Giner T, d Jozsi M. Complement factor H-antibodyassociated hemolytic uremic syndrome: pathogenesis, clinical presentation, and treatment. Semin Thromb Hemost. 2014;40:431-43.

73. Nester CM, Thomas CP. Atypical hemolytic uremic syndrome: what is it, how is it diagnosed, and how is it treated? Hematol-Am Soc Hemat. 2012;617-625. This review provides an overview of aHUS along with insights into new treatments.

74. Noris M, Galbusera M, Gastoldi S, Macor P, Banterla F, et al. Dynamics of complement activation in aHUS and how to monitor eculizumab therapy. Blood. 2014;124:1715-26.

75. Yoshioka K, Hino S, Takemura T, Maki S, Wieslander J, et al. Type IV collagen alpha 5 chain. Normal distribution and abnormalities in $\mathrm{X}$-linked Alport syndrome revealed by monoclonal antibody. Am J Pathol. 1994;144:986-96.

76. Gubler M, Levy M, Broyer M, Naizot C, Gonzales G, et al. Alport's syndrome. A report of 58 cases and a review of the literature. Am J Med. 1981;70:493-505. 
77. Barker DF, Hostikka SL, Zhou J, Chow LT, Oliphant AR, et al. Identification of mutations in the COL4A5 collagen gene in Alport syndrome. Science. 1990;248:1224-7.

78. Mochizuki T, Lemmink HH, Mariyama M, Antignac C, Gubler $\mathrm{MC}$, et al. Identification of mutations in the alpha 3(IV) and alpha 4(IV) collagen genes in autosomal recessive Alport syndrome. Nat Genet. 1994;8:77-81.

79. Feingold J, Bois E, Chompret A, Broyer M, Gubler MC, et al. Genetic heterogeneity of Alport syndrome. Kidney Int. 1985;27: 672-7.

80. van der Loop FT, Heidet L, Timmer ED, van den Bosch BJ, Leinonen A, et al. Autosomal dominant Alport syndrome caused by a COL4A3 splice site mutation. Kidney Int. 2000;58:1870-5.

81. Savige J, Rana K, Tonna S, Buzza M, Dagher H, et al. Thin basement membrane nephropathy. Kidney Int. 2003;64:1169-78.

82. Jais JP, Knebelmann B, Giatras I, De Marchi M, Rizzoni G, et al. Xlinked Alport syndrome: natural history and genotype-phenotype correlations in girls and women belonging to 195 families: a "European Community Alport Syndrome Concerted Action" study. J Am Soc Nephrol JASN. 2003;14:2603-10.

83. Savige J, Gregory M, Gross O, Kashtan C, Ding J, et al. Expert guidelines for the management of Alport syndrome and thin basement membrane nephropathy. J Am Soc Nephrol JASN. 2013;24: 364-75.

84. Gross O, Weber M, Fries JW, Muller GA. Living donor kidney transplantation from relatives with mild urinary abnormalities in
Alport syndrome: long-term risk, benefit and outcome. Nephrol Dial Transplant Off Publ Eur Dial Transplant Assoc Eur Ren Assoc. 2009;24:1626-30.

85. Pierides A, Voskarides K, Athanasiou Y, Ioannou K, Damianou L, et al. Clinico-pathological correlations in 127 patients in 11 large pedigrees, segregating one of three heterozygous mutations in the COL4A3/ COL4A4 genes associated with familial haematuria and significant late progression to proteinuria and chronic kidney disease from focal segmental glomerulosclerosis. Nephrol Dial Transplant Off Publ Eur Dial Transplant Assoc Eur Ren Assoc. 2009;24:2721-9.

86. Deltas C, Pierides A, Voskarides K. Molecular genetics of familial hematuric diseases. Nephrol Dial Transplant Off Publ Eur Dial Transplant Assoc Eur Ren Assoc. 2013;28:2946-60.

87. Ierino FL, Kanellis J. Thin basement membrane nephropathy and renal transplantation. Semin Nephrol. 2005;25:184-7.

88. Sakai K, Muramatsu M, Ogiwara H, Kawamura T, Arai K, et al. Living related kidney transplantation in a patient with autosomalrecessive Alport syndrome. Clin Transpl. 2003;17 Suppl 10:4-8.

89. MacDermot KD, Holmes A, Miners AH. Anderson-Fabry disease: clinical manifestations and impact of disease in a cohort of 60 obligate carrier females. J Med Genet. 2001;38:769-75.

90. Germain DP, Waldek S, Banikazemi M, Bushinsky DA, Charrow J, et al. Sustained, long-term renal stabilization after 54 months of agalsidase beta therapy in patients with Fabry disease. J Am Soc Nephrol JASN. 2007;18:1547-57. 